\title{
Combined analysis of effective Higgs portal dark matter models
}

\author{
Ankit Beniwal, ${ }^{a}$ Filip Rajec, ${ }^{a}$ Christopher Savage,${ }^{b c}$ Pat Scott, ${ }^{d}$ Christoph Weniger, ${ }^{e}$ \\ Martin White $^{a}$ and Anthony G. Williams ${ }^{* a}$ \\ ${ }^{a}$ ARC Center of Excellence for Particle Physics at the Terascale \& CSSM, Department of Physics, \\ University of Adelaide, Adelaide, South Australia 5005, Australia \\ ${ }^{b}$ Nordita, KTH Royal Institute of Technology and Stockholm University, Stockholm, Sweden \\ ${ }^{c}$ Department of Physics \& Astronomy, University of Utah, Salt Lake City, Utah, USA \\ ${ }^{d}$ Department of Physics, Imperial College London, Blackett Laboratory, Prince Consort Road, \\ London SW7 2AZ, United Kingdom \\ ${ }^{e}$ GRAPPA, University of Amsterdam, Science Park 904, 1090 GL Amsterdam, Netherlands \\ Email: ankit.beniwal@adelaide.edu.au, filip.rajec@adelaide.edu.au, \\ chris@savage.name, p.scotteimperial.ac.uk, c.wenigereuva.nl, \\ martin.white@adelaide.edu.au, anthony.williams@adelaide.edu.au \\ ORCID by initials: AB: 0000-0003-4849-0611, AGW: 0000-0002-1472-1592
}

\begin{abstract}
We perform a combined and extended analysis of effective scalar, vector, Majorana and Dirac fermion Higgs portal models of dark matter (DM). In these models, DM couples to the Standard Model (SM) Higgs boson via an operator of the form $\mathscr{O}_{\mathrm{DM}} H^{\dagger} H$. For the fermion models, we allow an admixture of the scalar $\bar{\psi} \psi$ and pseudoscalar $\bar{\psi} i \gamma_{5} \psi$ interaction terms. On each model, we impose constraints from the Planck measured DM relic density, LHC limits on the Higgs invisible branching ratio, indirect search limits from the WMAP7/Planck observations of the cosmic microwave background, a combined analysis of 15 dwarf spheroidal galaxies by Fermi-LAT and the upcoming Cherenkov Telescope Array (CTA) as well as the direct search limits from the LUX and projected XENON1T experiments. We find in line with previous studies that the direct search experiments will continue to exclude large portions of the model parameter space. Due to the momentum suppression of the direct search cross section in the case of a pure pseudoscalar interaction of a fermionic DM candidate, future indirect searches are the only class of experiments capable of probing the high mass range of the theory.
\end{abstract}

38th International Conference on High Energy Physics

3-10 August 2016

Chicago, USA

* Speaker. 


\section{Introduction}

The Standard Model (SM) of particle physics has been very successful at explaining all the non-gravitational interactions of the ordinary matter. However, it does not provide any viable candidates for Dark Matter (DM) which makes up 85\% of the total matter density in our Universe. Therefore, possible candidates for DM are looked for in theories that lie beyond the SM (BSM).

In the Higgs portal scenario, DM interacts with the SM particles via the exchange of a SM Higgs boson [1]. These models are motivated by the simplicity in the required BSM particle content and the operator $H^{\dagger} H$ being one of the two lowest-dimensional gauge-invariant operators in the SM. ${ }^{1}$

Here, we present a subset of our results in Ref. [2] where a complete description of the current and projected future limits on the effective scalar, vector, Majorana and Dirac fermion Higgs portal models is provided. On each model, we apply currently available constraints arising from the DM relic density, the Higgs invisible width, indirect and direct search experiments. For the indirect searches, our current limits are based on the WMAP 7-year observations of the cosmic microwave background (WMAP7) and a combined analysis of 15 dwarf galaxies by Fermi-LAT; for the projected future limits, we use the Planck polarization data, projected improvements in the Fermi-LAT observation of additional dwarf galaxies and prospects for the upcoming Cherenkov Telescope Array (CTA). Our direct detection limits are derived using the results from the XENON100 (2012) and LUX (2013) experiments.

\section{Models}

Assuming the DM fields are SM gauge singlets, we consider a scalar $(S)$, vector $\left(V_{\mu}\right)$, Majorana $(\chi)$ and Dirac $(\psi)$ fermion DM candidate. Lagrangians for the DM fields are ${ }^{2}$

$$
\begin{array}{r}
\mathscr{L}_{S}=\mathscr{L}_{\mathrm{SM}}+\frac{1}{2}\left(\partial_{\mu} S\right)\left(\partial^{\mu} S\right)-\frac{1}{2} \mu_{S}^{2} S^{2}-\frac{1}{4 !} \lambda_{S} S^{4}-\frac{1}{2} \lambda_{h S} S^{2} H^{\dagger} H, \\
\mathscr{L}_{V}=\mathscr{L}_{\mathrm{SM}}-\frac{1}{4} W_{\mu v} W^{\mu v}+\frac{1}{2} \mu_{V}^{2} V_{\mu} V^{\mu}-\frac{1}{4 !} \lambda_{V}\left(V_{\mu} V^{\mu}\right)^{2}+\frac{1}{2} \lambda_{h V} V_{\mu} V^{\mu} H^{\dagger} H, \\
\mathscr{L}_{\chi}=\mathscr{L}_{\mathrm{SM}}+\frac{1}{2} \bar{\chi}\left(i \not \gamma-\mu_{\chi}\right) \chi-\frac{1}{2} \frac{\lambda_{h \chi}}{\Lambda_{\chi}}\left(\cos \theta \bar{\chi} \chi+\sin \theta \bar{\chi} i \gamma_{5} \chi\right) H^{\dagger} H, \\
\mathscr{L}_{\psi}=\mathscr{L}_{\mathrm{SM}}+\bar{\psi}\left(i \not \gamma-\mu_{\psi}\right) \psi-\frac{\lambda_{h \psi}}{\Lambda_{\psi}}\left(\cos \theta \bar{\psi} \psi+\sin \theta \bar{\psi} i \gamma_{5} \psi\right) H^{\dagger} H,
\end{array}
$$

where $\mathscr{L}_{\mathrm{SM}}$ is the SM Lagrangian, $W_{\mu \nu} \equiv \partial_{\mu} V_{v}-\partial_{\nu} V_{\mu}$ is the field strength tensor of the vector field $V_{\mu}$ and $H$ is the SM Higgs doublet. In the fermion models, $\cos \theta=1(0)$ leads to a pure scalar (pseudoscalar) interaction between the fermion DM and the SM Higgs boson.

After electroweak symmetry breaking (EWSB), the SM Higgs doublet reduces in the unitary gauge to $H=\left(0, v_{0}+h\right)^{T} / \sqrt{2}$, where $h$ is the physical SM Higgs field and $v_{0}=246.22 \mathrm{GeV}$ is its vacuum expectation value (VEV). As a result, the $H^{\dagger} H$ term generates mass and interaction terms for the DM fields $X \in\left(S, V_{\mu}, \chi, \psi\right)$.

\footnotetext{
${ }^{1}$ The other is the hypercharge field strength tensor $B_{\mu v}$.

${ }^{2}$ Since DM candidates are required to be stable on cosmological time scales, an assumed $\mathbb{Z}_{2}$ symmetry: $X \rightarrow-X$ for $X \in\left(S, V_{\mu}, \chi, \psi\right)$ is imposed on the DM fields.
} 
When $\sin \theta \neq 0$ in the fermion models, non-mass-type contributions appear which are purely quadratic in the DM fields. To define a physical real mass, a chiral rotation is performed on the post-EWSB fermion fields. Following the results outlined in the Appendix of Ref. [2], this ultimately reduces to the substitution: $\mu_{\chi}\left(\mu_{\psi}\right) \rightarrow m_{\chi}\left(m_{\psi}\right)$ and $\theta \rightarrow \xi \equiv \theta+\alpha$ where $m_{\chi}\left(m_{\psi}\right)$ are the physical masses of the Majorana (Dirac) fermion DM. ${ }^{3}$

\section{Constraints}

\subsection{Relic density}

We compute the model relic density, the annihilation cross section $\sigma v_{\text {rel }}$ and the gamma-ray yields per annihilation using the publicly available software micrOMEGAs 3.6.9.2 [3]. ${ }^{4}$ The computed model relic density is matched with the Planck measured central value, $\Omega_{\mathrm{DM}} h^{2}=0.1199$ [4]. This places a constraint on the Higgs portal coupling for each DM mass.

As there is a strong possibility of a multicomponent dark sector, we define a relic abundance parameter $f_{\text {rel }} \equiv \Omega_{X} / \Omega_{\text {DM }}$ to explore scenarios when the model $X$ makes up $100 \%\left(f_{\text {rel }}=1\right), 10 \%$ $(0.1)$ and $1 \%(0.01)$ of the total DM density.

\subsection{Higgs invisible width}

The decay $h \rightarrow \bar{X} X$ for $X \in\left(S, V_{\mu}, \chi, \psi\right)$ is kinematically allowed when $m_{X}<m_{h} / 2$. This contributes to the SM Higgs invisible width $\left(\Gamma_{\text {inv }}\right)$.

To impose the Higgs invisible width constraint, we use the results of Ref. [5] where an upper limit of $19 \%$ on the SM Higgs invisible branching ratio $B R(h \rightarrow \bar{X} X)$ at $2 \sigma$ C.L. is obtained after combining fits from all the Higgs production and decay channels probed by ATLAS, CMS and the Tevatron. Using the upper limit on $B R(h \rightarrow \bar{X} X)$ and the expressions for $\Gamma_{\text {inv }}$ in our models, an upper limit is placed on the Higgs portal coupling for DM masses up to $m_{h} / 2$.

\subsection{Indirect detection}

We impose the indirect search limits using a combined log-likelihood function. The current indirect search limits come from a 7-year observation of the cosmic microwave background (CMB) by WMAP [6] and a combined analysis of 15 dwarf galaxy observations by Fermi-LAT [7]. The projected future limits are obtained from the Planck polarization data, projected improvements in the Fermi-LAT sensitivity towards observation of additional dwarf galaxies and prospects for the planned CTA [8] (for more details, see Ref. [2]).

All the indirect search signals are scaled by $f_{\text {rel }}^{2}=\left(\Omega_{X} / \Omega_{\mathrm{DM}}\right)^{2}$, resulting in suppressed signals when the model $X$ constitutes only a fraction of the total DM density.

\footnotetext{
${ }^{3}$ Due to the chiral rotation, the post-EWSB parameters $m_{\chi, \psi}, \cos \xi$ and $\sin \xi$ are functions of the pre-EWSB parameters $\mu_{\chi, \psi}, \cos \theta$ and $\sin \theta$ (see Ref. [2] for more details).

${ }^{4}$ The quartic self-couplings $\lambda_{S, V}$ can be ignored throughout the analysis since they play no observable role in the DM phenomenology.
} 


\subsection{Direct detection}

Our Higgs-mediated scalar and/or pseudoscalar couplings lead to a spin independent (SI) WIMP-nucleon interaction. For the fermion models when $\sin \xi \neq 0$, the SI cross section is suppressed by $q^{2} / 4 m_{\chi, \psi}^{2}$, where $q$ is the momentum transfer of the order of few MeVs.

We impose the direct limits from the LUX [9] experiment using a generalized version of the LUXCalc 1.0.1 package [10]. The projected limits from the XENON1T experiment are based on scaling the $90 \%$ C.L. from XENON100 [11] by an exposure $\varepsilon=100$. Limits from both experiments are appropriately scaled by $f_{\text {rel }}=\Omega_{X} / \Omega_{\mathrm{DM}}$ to account for the possibility of a multicomponent dark sector.

\section{Results}

Combined limits from indirect searches in the $\left(m_{\psi}, \lambda_{h \psi} / \Lambda_{\psi}\right)$ plane are shown in Fig. 1 for $\cos \xi=1$ (left) and $\cos \xi=0$ (right). ${ }^{5}$ The grey and pink shaded regions are excluded respectively by the DM relic density and the Higgs invisible width constraints. The green shaded region where $\lambda_{h \psi} / \Lambda_{\psi}>4 \pi / 2 m_{\psi}$ leads to a break down of the EFT approximation in DM annihilations.

When the interaction is pure scalar $(\cos \xi=1)$, the annihilation cross section $\sigma v_{\text {rel }}$ is $v^{2}$ suppressed where $v \sim 10^{-3} c$ is the DM relative speed in our local halo. Consequently, the resulting limits are weak and no exclusion of the parameter space is possible. On the contrary, when the interaction is pure pseudoscalar $(\cos \xi=0)$, the velocity suppression in $\sigma v_{\text {rel }}$ is lifted and nontrivial indirect search limits are obtained. In this case, the projected future indirect searches will be able to exclude Dirac fermion masses up to $\sim 74 \mathrm{GeV}$ for the Einasto profile and between $\sim 166 \mathrm{GeV}$ and $1.3 \mathrm{TeV}$ for a contracted generalized NFW profile, if $\psi$ makes up all of the DM.
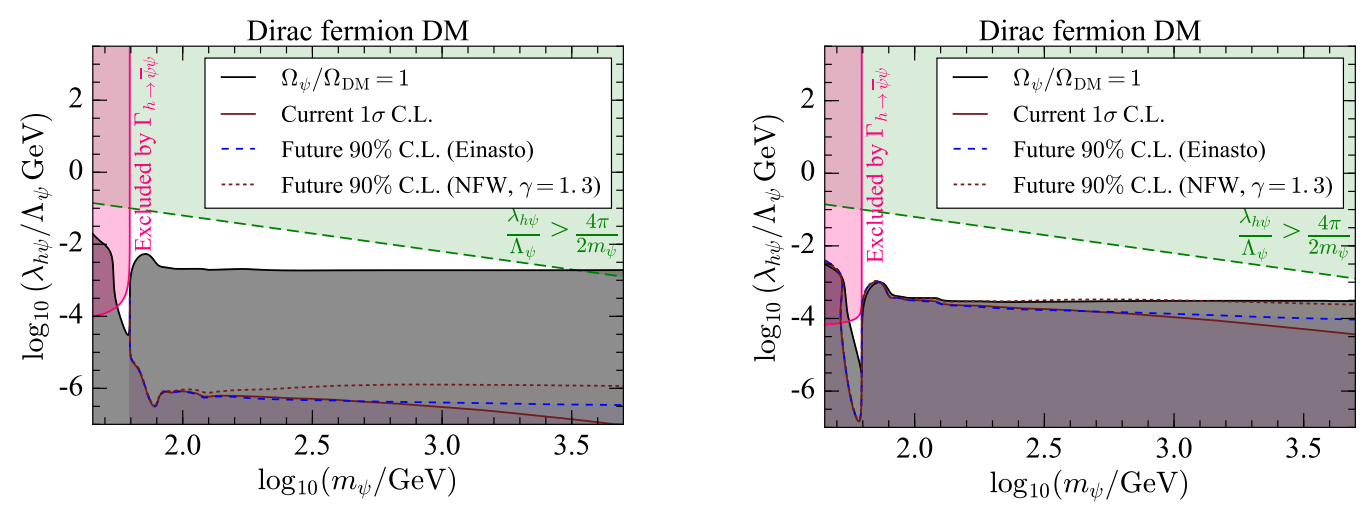

Figure 1: Combined indirect search limits in the $\left(m_{\psi}, \lambda_{h \psi} / \Lambda_{\psi}\right)$ plane for $\cos \xi=1$ (left) and 0 (right). The grey (pink) shaded regions are excluded by the DM relic density (Higgs invisible width) constraints. The green shaded region is where the EFT approximation in DM annihilations break down. Values of $\lambda_{h \psi} / \Lambda_{\psi}$ below the current $1 \sigma$ C.L. (brown solid) curve are excluded at more than $1 \sigma$ C.L. whereas the regions below the future 90\% C.L. curve with the Einasto (blue dashed) profile and contracted NFW (brown dotted) profile will be excluded in the near future.

\footnotetext{
${ }^{5}$ As mentioned in the introduction, we only present a subset of our results to meet the required page limit. Specifically, we present the results for the Dirac fermion model when $\cos \xi=1$ and 0 . For interested readers, see our full set of model results in Ref. [2].
} 

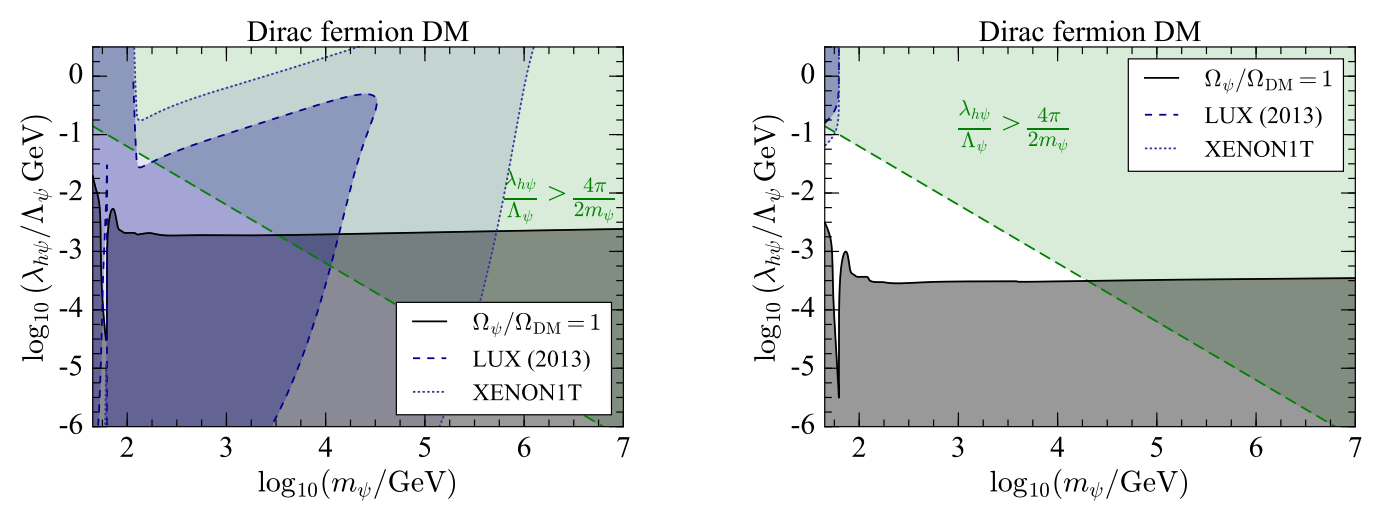

Figure 2: Direct search limits in the $\left(m_{\psi}, \lambda_{h \psi} / \Lambda_{\psi}\right)$ plane for $\cos \xi=1$ (left) and 0 (right). The grey and green shaded regions are same as in Fig. 1. The regions excluded by the LUX (projected XENON1T) experiments are delineated with the blue dashed (blue dotted) curves and dark (light) shadings.

The direct search limits in the $\left(m_{\psi}, \lambda_{h \psi} / \Lambda_{\psi}\right)$ plane are shown in Fig. 2 for $\cos \xi=1$ (left) and 0 (right). The LUX and projected XENON1T limits in the green shaded are subject to UV corrections as they are scaled by $f_{\text {rel }}=\Omega_{\psi} / \Omega_{\mathrm{DM}}$. Consequently, the resulting limits on $m_{\psi}$ above $\sim 3.3 \mathrm{TeV}(\sim 19.7) \mathrm{TeV}$ when $\cos \xi=1$ ( 0 ) cannot be guaranteed to keep the EFT approximation in DM annihilations valid, if $\psi$ makes up all of the DM.

When $\cos \xi=0$, the SI cross section is momentum suppressed by a factor of $q^{2} / 4 m_{\chi, \psi}^{2}$, resulting in a small number of direct search event rates. Indirect search experiments (the right panel in Fig. 1) are our only hope of probing the higher DM mass range in this case.

The momentum suppression of the SI cross section is lifted when $\cos \xi=1$. This results in a significant direct search event rate. The LUX experiment excludes Dirac masses up to $\sim$ 3.3 TeV. When the limits from the DM relic density, the Higgs invisible width and the projected XENON1T experiment are combined, the entire low mass region up to $m_{h} / 2$ will be excluded. ${ }^{6}$ The projected XENON1T experiment will also reach enough sensitivity to exclude TeV-scale Dirac fermion masses, if $\psi$ makes up all of the DM.

\section{Conclusions}

We have performed an updated and combined analysis of effective scalar, vector, Majorana and Dirac fermion Higgs portal models of dark matter in Ref. [2]. For the fermion models, the DM-SM Higgs boson coupling was chosen to be either pure scalar, pure pseudoscalar or an equal mix between the scalar and pseudoscalar terms. The presence of the pseudoscalar term and the requirement of a quadratic DM mass term led us to redefine the post-EWSB fermion fields via a chiral rotation.

The combined constraints on the DM relic density and the Higgs invisible width excludes most of the low mass parameter space. When the projected XENON1T limits are imposed, low Dirac fermion masses will be entirely excluded. Although the current and projected future indirect

\footnotetext{
${ }^{6}$ In contrast, parts of the same region are allowed in the scalar, vector and Majorana fermion DM models (see Figs. 4, 7 and 13 in Ref. [2] for a comparison).
} 
search limits are weak in comparison with the direct search limits, they are nevertheless important (especially for the fermion models when $\cos \xi=0$ ).

\section{Acknowledgments}

We thank Csaba Balazs, Jim Cline, Alexander Pukhov, Geneviève Bélanger and Andre Scaffidi for useful discussions. The work of A. B., F. R. and A. G. W. is supported by the ARC Centre of Excellence for Particle Physics at the Terascale (CoEPP) (CE110001104) and the Centre for the Subatomic Structure of Matter (CSSM). M. W. is supported by the Australian Research Council Future Fellowship FT140100244. P. S. is supported by the STFC (Grants No. ST/K00414X/1 and No. ST/N000838/1).

\section{References}

[1] V. Silveira and A. Zee, Scalar phantoms, Phys. Lett. 161B 136, (1985); C. P. Burgess, M. Pospelov, and T. ter Veldhuis, The Minimal model of nonbaryonic dark matter: A singlet scalar, Nucl. Phys. B619 709-728, (2001), [hep-ph/ 0011335 ].

[2] A. Beniwal, F. Rajec, C. Savage, P. Scott, C. Weniger, M. White and A. G. Williams, Combined analysis of effective Higgs portal dark matter models, Phys. Rev. D 93, 115016, (2016), [arXiv:1512.06458].

[3] G. Belanger, F. Boudjema, A. Pukhov, and A. Semenov, micrOMEGAs_3: A program for calculating dark matter observables, Comput. Phys. Commun. 185 960-985, (2014), [arXiv:1305. 0237].

[4] Planck Collaboration, P. Ade et al., Planck 2013 results. XVI. Cosmological parameters, Astron.Astrophys. 571 A16, (2014), [arXiv:1303.5076].

[5] G. Belanger, B. Dumont, U. Ellwanger, J. Gunion, and S. Kraml, Global fit to Higgs signal strengths and couplings and implications for extended Higgs sectors, Phys. Rev. D 88 075008, (2013), [arXiv:1306.2941].

[6] J. M. Cline and P. Scott, Dark Matter CMB Constraints and Likelihoods for Poor Particle Physicists, JCAP 1303 044, (2013), [arXiv: 1301 . 5908]. [Erratum: JCAP 1305, E01 (2013)].

[7] Fermi-LAT, M. Ackermann et al., Searching for Dark Matter Annihilation from Milky Way Dwarf Spheroidal Galaxies with Six Years of Fermi Large Area Telescope Data, Phys. Rev. Lett. 115 231301, (2015), [arXiv:1503.02641].

[8] H. Silverwood, C. Weniger, P. Scott, and G. Bertone, A realistic assessment of the CTA sensitivity to dark matter annihilation, J. Cosmol. Astropart. Phys. 1503 055, (2015), [arXiv: 1408 .4131].

[9] LUX Collaboration, D. Akerib et al., First Results from the LUX Dark Matter Experiment at the Sanford Underground Research Facility, Phys. Rev. Lett. 112 091303, (2014),

[arXiv: 1310.8214$]$.

[10] C. Savage, A. Scaffidi, M. White, and A. G. Williams, LUX likelihood and limits on spin-independent and spin-dependent WIMP couplings with LUXCalc, Phys. Rev. D 92 103519, (2015), [arXiv: 1502.02667].

[11] XENON100 Collaboration, E. Aprile et al., Dark Matter Results from 225 Live Days of XENON100 Data, Phys. Rev. Lett. 109 181301, (2012), [arXiv:1207.5988]. 